\title{
Research
}

\section{Frequency of Cases of Genitourinary Tuberculosis Hospital De Clínicas Gestión 2000 to 2019 La Paz, Bolivia}

\author{
Paz Cabrera Luz Gloria ${ }^{1,2^{*}}$, Vélez Gisbert Gróver ${ }^{1}$ \\ ${ }^{1}$ Department of Urology, Hospital de Clínicas. La Paz, Bolivia \\ ${ }^{2}$ Department of Urology, Municipal Hospital La Portada. La Paz, Bolivia
}

*Correspondence to: Paz Cabrera Luz Gloria; Department of Urology, Hospital de Clínicas. La Paz, Bolivia; E-mail: luz_gloria_urologia@hotmail.com Received: April 14 ${ }^{\text {th }}, 2020$; Accepted: June 20 $0^{\text {th }}, 2020$; Published: June 27 ${ }^{\text {th }}, 2020$

Citation: Gloria LCP, Gisbert VG, Grover. Frequency of cases of Genitourinary Tuberculosis Hospital De Clínicas gestión 2000 to 2019 La Paz, Bolivia. Urology Open A Open J. 2020; I(1): 1-4.

\begin{abstract}
Introduction

The urogenital tract is one of the most frequent locations of extrapulmonary tuberculosis, but it is not a very common finding in clinical practice. According to the WHO in 2015, 10.4 million people fell ill with tuberculosis, which is one of the top 10 global causes of mortality. Extrapulmonary tuberculosis is estimated to represent 15 to $25 \%$ of all tuberculosis patients, and of these, the renal location is the most frequent.

\section{Methods}

We publish the present study, because tuberculosis is one of the endemic infectious diseases in Bolivia, and its study is essential. It should be mentioned that in Bolivia, as part of the immunization scheme, all newborns receive the BCG vaccine. All patients with antituberculosis treatment initiation, registered in the Notebook of the National Program for Tuberculosis Control, from January 2000 to December 2019 (19 years old) have been included with the diagnosis of genitourinary tuberculosis. Under the responsible Units: Urology and Epidemiology of the Hospital de Clínicas, in the City of La Paz
\end{abstract}

\section{Results}

The frequency of cases of genitourinary tuberculosis in the Hospital of Clinics of the City of La Paz in 2000 to 2019 procedures is low and is correlated with bibliographic data. 5,025 patients with tuberculosis were diagnosed in 2000 to 2019 procedures of which 1,507 patients (30\%) had pulmonary tuberculosis. 3,518 patients $(70 \%)$ with extrapulmonary tuberculosis of the total of patients with extrapulmonary tuberculosis, 845 patients were diagnosed with urogenital tuberculosis of which 625 patients were diagnosed with renal tuberculosis, 53 patients with bladder tuberculosis, 19 patients with prostate tuberculosis, 81 patients with epididymal tuberculosis and 67 patients with genital (adnexal) tuberculosis.

\section{Conclusions}

Over time, infectious contagious pathologies have become relevant. Mainly due to the living conditions, housing, nutrition, and coexistence of pathologies that depress the immune response. The difficulty of obtaining data prior to the initiation of tuberculosis treatment and monitoring of fertility in this group of patients involves a significant economic cost since the highest percentages of diagnosed patients are of reproductive age.

Keywords: Extrapulmonary tuberculosis; Genitourinary tuberculosis; Renal tuberculosis.

\section{INTRODUCTION}

The urogenital tract is one of the most frequent locations of extrapulmonary tuberculosis, but it is not a very common finding in clinical practice. According to the WHO in 2015, 10.4 million people fell ill with tuberculosis, which is one of the top 10 global causes of mortality. Extrapulmonary tuberculosis is estimated to represent $15 \%$ to $25 \%$ of all tuberculosis patients, and of these, the renal location is the most frequent. ${ }^{1}$ 


\section{History}

Tuberculosis has been observed for more than 7,000 years. Hippocrates described "phthisis" as a pathology that increased in the winter and led to wasting, and his treatment methods were followed for more than 1,500 years. Koch in 1882 discovered the Bacillus that bears his name. ${ }^{2}$

\section{Etiology}

Koch's Bacillus (BK) or Mycobacterium tuberculosis is the bacillus that causes $99 \%$ of cases of human tuberculosis. Its characteristics are. ${ }^{2}$

- Aerobic, resistant acid, being able to multiply within the alveoli.

- Slow growth (multiplies in 20 to 24 hours).

- It develops at a pH of 6.8 - 7.2.

- Stain with Ziehl-Neelsen staining

Classification of Mycobacteria

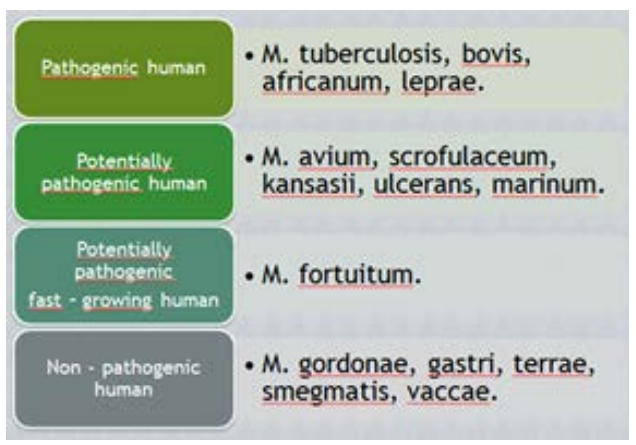

\section{Etiopathogenesis}

Primary focus occurs within macrophages, which have no ability to control and the spread, by the lymphatic route and then by blood route that stops around the fourth week. The primary focus may be on the lungs or on dormant lymph node nuclei. ${ }^{3}$

The kidney is infected hematogenously and its dissemination can be lymphatic or canalicular. BK can be eliminated in the urine without pyuria or symptoms of bladder irritation.

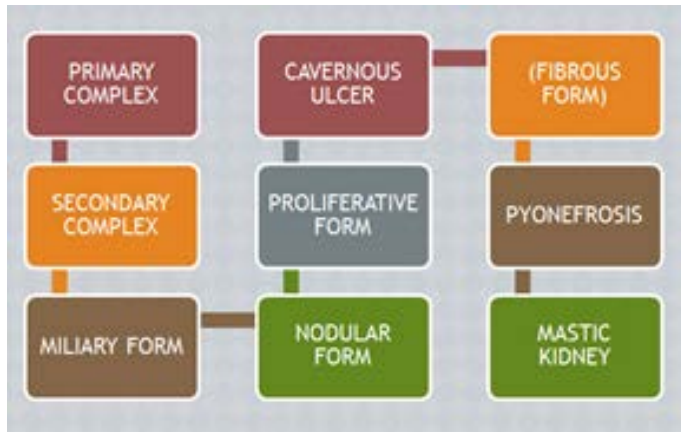

\section{Statistics}

In Latin America, Bolivia, along with Peru, has the highest incidence. For 2000 the incidence of tuberculosis in all its forms was calculated in Bolivia at 112 and for pulmonary tuberculosis BAAR (+) in 78 cases per 1,00,000 inhabitants. 8,000 new cases are diagnosed each year. From 2002 to $2004,13 \%$ to $15 \%$ of extrapulmonary tuberculosis cases were reported. ${ }^{1}$
In Bolivia, by decree, it has been determined that on March 24 of each year the Day to Fight Tuberculosis is celebrated. $83 \%$ of tuberculosis patients come to a cure at the end of treatment. Since 2007, a program has been implemented in the prisons of Bolivia for the detection and diagnosis of tuberculosis (since $69 \%$ of the diagnosed patients corresponded to incarcerated patients).

\section{Clinic}

The symptoms are intermittent and variable in intensity and duration. $20 \%$ of patients are initially asymptomatic. The most frequent age is in young adults (18 to 30 years old). Syphilis has traditionally been defined as the "great simulator" of infectious contagious diseases, however, tuberculosis is just as protein-like in its clinical manifestations. This is due, among other causes, to three important factors. The first is its capacity for hematogenous and lymphatic dissemination. This gives it the ability to alter the morphology and function of virtually all the organs of the human body, even many years after the first infection, due to its ability to remain dormant in them. Secondly, as it is an intracellular infection, it generates an immune response in which the release of proinflammatory cytokines occasionally prevails, triggering processes that can simulate autoimmune phenomena. And finally, their associations with significant comorbidities that can be both conditioning factors for tuberculosis and conditioned by it and that have their own semiological richness. ${ }^{4-7}$

The initial focus is on the renal cortex, characterized by the destruction caused by the inflammatory reaction due to the immune manifestation of delayed hypersensitivity. The process progresses slowly in 15 to 20 years can destroy the entire kidney. It begins by committing an area to later spread to neighboring regions. It forms small nodules or tubers that soften and necrose (ulcer cavernous lesions), coming together to form small caverns that progressively increase in volume.

They can be up to the size of a chestnut and contain a cheesy substance that in some cases is calcified by calcium salt deposits, forming the kidney type "mastic", visible to X-rays. Genitourinary tuberculosis must be understood as a disease that encompasses the entire genitourinary system; genital tuberculosis is generally secondary to urinary tuberculosis that, via the canalicular or lymphatic route, reaches the epididymis, testis or prostate. The reaction of defense mechanisms produces fibrous tissue that causes capillary or pyeloureteral junction stenosis, since the lesions extend throughout the mucosa of the renal pelvis, ureter, and bladder, covering it with tubercles [Figure 1].

Figure 1. Operative part Kidney mastic (caseum and renal caverns identified).
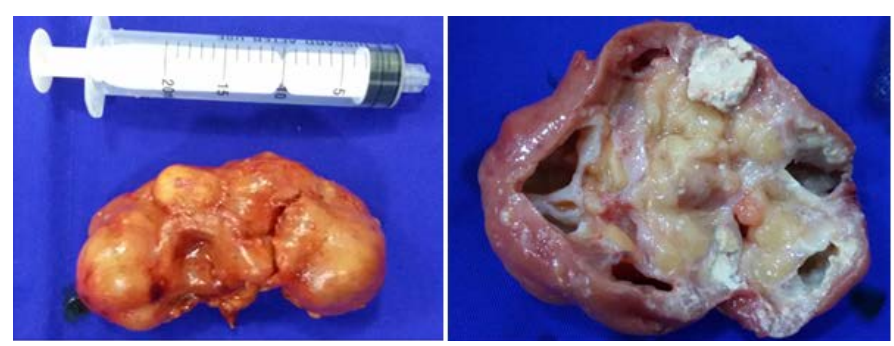

Individual objectives of anti-tuberculosis treatment

Produce smear and/or culture negativity in the shortest possible time. Ensure adherence (more than $80 \%$ of doses) and completion of therapy to prevent resistance and ensure healing. Recognize side effects early. 


\section{Antitubercular drugs in Bolivia}

The national tuberculosis program uses seven basic drugs. ${ }^{1}$

Bactericides: isoniazid (H), rifampicin (R), streptomycin (S), and pyrazinamide (Z).

Bacteriostatic: Ethambutol (E), Thioacetazone (T) and Ethionamide (ETA)

\section{METHODS}

We publish the present study, because tuberculosis is one of the endemic infectious diseases in Bolivia, and its study is essential. It should be mentioned that in Bolivia, as part of the immunization scheme, all newborns receive the BCG vaccine. All patients with antituberculosis treatment initiation, registered in the Notebook of the National Program for Tuberculosis Control, from January 2000 to December 2019 (19 years old) have been included. With the diagnosis of genitourinary tuberculosis. Under the responsible Units: Urology and Epidemiology of the Hospital de Clínicas, in the City of La Paz.

\section{General Purpose}

To know the frequency of cases of urogenital tuberculosis in the Hospital of Clinics of the City of La Paz in the steps from 2000 to 2019.

\section{Specific Objectives}

To know the number of patients diagnosed with tuberculosis in its different forms of presentation.

Know the number of patients diagnosed with extrapulmonary tuberculosis and determine how many of them had urogenital tuberculosis.

\section{Research Design}

General study topic: Study of urological pathology.

Specific study topic: Study of Tuberculous pathology (Renal Tuberculosis).

Research type: Analytical. Cross. Retrospective.

\section{Data Measurement Method}

Source: Primary.

Instrument: Patient Registration Notebook. National tuberculosis control program.

Data processing: Manual.

\section{Population Unit- Place}

Spatial delimitation: Responsible units: Urology and Epidemiology of the Hospital de Clínicas, in the City of La Paz.

Temporary delimitation: January 2000 to December 2019. (19 years old).

Sample size. Unit: Patients included in the National Tuberculosis Control Program.

Inclusion criteria: All patients diagnosed with urogenital tuberculosis diagnosed by different methods, in the different units of the Hospital de Clínicas that entered the Notebook of the National Program for Tuberculosis Control.
Exclusion criteria: Patients who did not have complementary studies to diagnose tuberculosis pathology. Patients who were not registered in the notebook of the National Program for Tuberculosis Control.

Variables: Total of patients. Groups by gender. Forms of presentation of tuberculosis: pulmonary, extrapulmonary: urogenital. Forms of presentation of urogenital tuberculosis: renal, bladder, prostate, epididymal, and genital. Diagnostic methods.

\section{RESULTS}

The frequency of cases of genitourinary tuberculosis in the Hospital of Clinics of the City of La Paz in 2000 to 2019 procedures is low and is correlated with bibliographic data. 5,025 patients with tuberculosis were diagnosed in 2000 to 2019 procedures. Of which 1,507 patients (30\%) had pulmonary tuberculosis, 3,518 patients (70\%) had extrapulmonary tuberculosis. Of the total of patients with extrapulmonary tuberculosis, 845 patients were diagnosed with urogenital tuberculosis. Of which 625 patients were diagnosed with renal tuberculosis, 53 patients with bladder tuberculosis, 19 patients with prostate tuberculosis, 81 patients with epididymal tuberculosis, and 67 patients with genital (adnexal) tuberculosis [Table 1-4].

Table 1. Number of patients diagnosed with Tuberculosis per year

\begin{tabular}{|c|c|c|c|}
\hline Year & Total & Women & Men \\
\hline 2000 & 185 & 83 & 102 \\
\hline 2001 & 198 & 88 & 110 \\
\hline 2002 & 240 & 98 & 142 \\
\hline 2003 & 252 & 104 & 148 \\
\hline 2004 & 276 & 92 & 184 \\
\hline 2005 & 288 & 127 & 161 \\
\hline 2006 & 245 & 107 & 138 \\
\hline 2007 & 310 & 119 & 191 \\
\hline 2008 & 254 & 122 & 132 \\
\hline 2009 & 278 & 104 & 174 \\
\hline 2010 & 232 & 91 & 141 \\
\hline 2011 & 275 & 108 & 167 \\
\hline 2012 & 223 & 87 & 136 \\
\hline 2013 & 240 & 93 & 147 \\
\hline 2014 & 243 & 67 & 176 \\
\hline 2015 & 224 & 55 & 169 \\
\hline 2016 & 241 & 98 & 143 \\
\hline 2017 & 272 & 93 & 179 \\
\hline 2018 & 289 & 106 & 183 \\
\hline 2019 & 260 & 64 & 319 \\
\hline TOTAL & 5025 & & \\
\hline & & 1906 & \\
\hline
\end{tabular}

Table 2. Forms of presentation of Tuberculosis: Pulmonary and extrapulmonary

\begin{tabular}{|c|c|c|}
\hline Year & TB PULMONARY & TB EXTRAPULMONARY \\
\hline 2000 & 73 & 128 \\
\hline 2001 & 72 & 156 \\
\hline 2002 & 88 & 152 \\
\hline 2003 & 74 & 178 \\
\hline 2004 & 67 & 169 \\
\hline 2005 & 77 & 211 \\
\hline 2006 & 99 & 166 \\
\hline 2007 & 85 & 185 \\
\hline 2008 & 78 & 194 \\
\hline
\end{tabular}




\begin{tabular}{|c|c|c|}
\hline 2009 & 73 & 149 \\
\hline 2010 & 76 & 168 \\
\hline 2011 & 85 & 209 \\
\hline 2012 & 72 & 177 \\
\hline 2013 & 67 & 185 \\
\hline 2014 & 75 & 139 \\
\hline 2015 & 57 & 139 \\
\hline 2016 & 55 & 192 \\
\hline 2017 & 79 & 209 \\
\hline 2018 & 68 & 193 \\
\hline 2019 & 87 & 190 \\
\hline TOTAL & 1507 & 3518 \\
\hline
\end{tabular}

Table 3. Forms of presentation of extrapulmonary tuberculosis: Urogenital

\begin{tabular}{|c|c|c|}
\hline Year & TB EXTRAPULMONARY & UROGENITAL \\
\hline 2000 & 128 & 33 \\
\hline 2001 & 156 & 45 \\
\hline 2002 & 152 & 55 \\
\hline 2003 & 178 & 40 \\
\hline 2004 & 169 & 36 \\
\hline 2005 & 211 & 44 \\
\hline 2006 & 166 & 33 \\
\hline 2007 & 185 & 1 \\
\hline 2008 & 194 & 39 \\
\hline 2009 & 149 & 44 \\
\hline 2010 & 168 & 36 \\
\hline 2011 & 209 & 41 \\
\hline 2012 & 177 & 43 \\
\hline 2013 & 185 & 41 \\
\hline 2014 & 139 & 55 \\
\hline 2015 & 168 & 41 \\
\hline 2016 & 192 & 47 \\
\hline 2017 & 209 & 48 \\
\hline 2018 & 193 & 41 \\
\hline 2019 & 190 & 44 \\
\hline TOTAL & 3518 & 845 \\
\hline
\end{tabular}

Table 4. Forms of presentation of Urogenital Tuberculosis

\begin{tabular}{|c|c|c|c|c|c|c|}
\hline Year & UROGENITAL & KIDNEY & BLADDER & PROSTATE & EPIDIDYMIS & GENITAL \\
\hline 2000 & 33 & 30 & 2 & 0 & 1 & 0 \\
\hline 2001 & 45 & 31 & 4 & 2 & 2 & 6 \\
\hline 2002 & 55 & 44 & 3 & 0 & 3 & 5 \\
\hline 2003 & 40 & 33 & 1 & 2 & 1 & 3 \\
\hline 2004 & 36 & 28 & 2 & 0 & 2 & 4 \\
\hline 2005 & 44 & 32 & 2 & 1 & 5 & 4 \\
\hline 2006 & 33 & 26 & 1 & 1 & 4 & 1 \\
\hline 2007 & 41 & 33 & 2 & 2 & 2 & 2 \\
\hline 2008 & 39 & 32 & 4 & 0 & 1 & 2 \\
\hline 2009 & 44 & 31 & 3 & 0 & 6 & 4 \\
\hline 2010 & 36 & 23 & 3 & 1 & 6 & 4 \\
\hline 2011 & 41 & 30 & 4 & 1 & 1 & 5 \\
\hline 2012 & 43 & 34 & 2 & 2 & 2 & 3 \\
\hline 2013 & 39 & 28 & 3 & 0 & 4 & 4 \\
\hline 2014 & 55 & 35 & 4 & 3 & 8 & 5 \\
\hline 2015 & 41 & 33 & 3 & 1 & 10 & 4 \\
\hline 2016 & 47 & 29 & 3 & 1 & 10 & 4 \\
\hline
\end{tabular}

\begin{tabular}{|c|c|c|c|c|c|c|}
\hline 2017 & 48 & 34 & 2 & 0 & 9 & 3 \\
\hline 2018 & 41 & 28 & 3 & 0 & 6 & 4 \\
\hline 2019 & 44 & 31 & 2 & 1 & 7 & 3 \\
\hline TOTAL & 845 & 625 & 53 & 19 & 81 & 67 \\
\hline
\end{tabular}

\section{DISCUSSION}

In Bolivia, to speak of urogenital tuberculosis is to relate this concept generally to specific organs such as the kidneys. But in the same way, it is relevant to mention the cases of genital tuberculosis, in our environment the BCG vaccine is part of the immunization scheme but even so, cases continue to be seen and they do not decrease, more to the contrary they remain in a frequent exponential number.

\section{CONFLICTS OF INTEREST}

None.

\section{REFERENCES}

1. Manual de Normas Técnicas en Tuberculosis. Ministerio de Salud. La Paz - Bolivia. 2017.

2. Simon HB, Weinstein AJ, Pasternak MS, et al. Genitourinary Tuberculosis. Am J Med. 1977; 63(3): 410-420. doi: 10.1016/00029343(77)90279-0

3. Eastwood JB, Corbishley CM, Grang JM. Tuberculosis and the Kidney. J Am SocNephrol. 2001; 12(6): 1307-1314.

4. Lely Solari. Tuberculosis: Diversas Manifestaciones De Una Misma enfermedad. Rev Peru Med Exp Salud Pública. 2099; 26(3): 405-407.

5. Vargas R. Avances En La Epidemiología De La Tuberculosis. Rev. Per. Tuberculosis. Enfermedades Respiratorias. 1975; 35: 7-41.

6. Actas Hispanoamericana de Patología. VIII Congreso Virtual Hispanoamericano de Anatomía Patológica. Octubre de 2006.

7. Cardozo ME. Epidemiologia, Cuadro Clinico, Diagnóstico, Tratamiento y Prevención de la Tuberculosis. Instituto de Medicina Tropical. Pedro Kuori. La Habana, Cuba. 2006. 